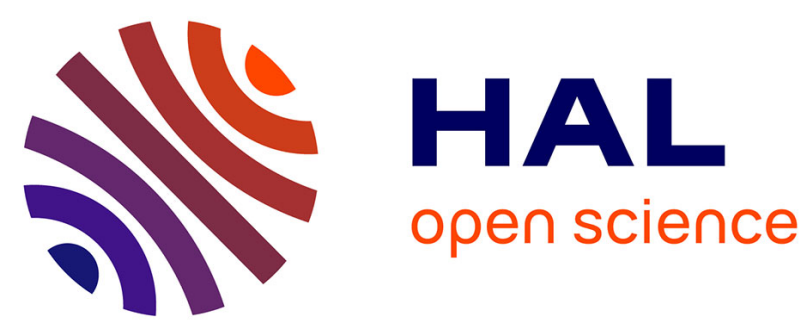

\title{
Impact of angiotensin-converting enzyme inhibitors or receptor blockers on post-ICU discharge outcome in patients with acute kidney injury
}

Etienne Gayat, Alexa Hollinger, Alain Cariou, Nicolas Deye, Antoine Vieillard-Baron, Samir Jaber, Benjamin Chousterman, Qin Lu, Pierre François Laterre, Xavier Monnet, et al.

\section{To cite this version:}

Etienne Gayat, Alexa Hollinger, Alain Cariou, Nicolas Deye, Antoine Vieillard-Baron, et al.. Impact of angiotensin-converting enzyme inhibitors or receptor blockers on post-ICU discharge outcome in patients with acute kidney injury. Intensive Care Medicine, 2018, 44 (5), pp.598-605. 10.1007/s00134018-5160-6 . hal-01893051

\section{HAL Id: hal-01893051 \\ https://hal.umontpellier.fr/hal-01893051}

Submitted on 15 Jan 2020

HAL is a multi-disciplinary open access archive for the deposit and dissemination of scientific research documents, whether they are published or not. The documents may come from teaching and research institutions in France or abroad, or from public or private research centers.
L'archive ouverte pluridisciplinaire HAL, est destinée au dépôt et à la diffusion de documents scientifiques de niveau recherche, publiés ou non, émanant des établissements d'enseignement et de recherche français ou étrangers, des laboratoires publics ou privés. 


\section{Impact of angiotensin-converting}

\section{enzyme inhibitors or receptor blockers on post-ICU discharge outcome in patients with acute kidney injury}

Etienne Gayat ${ }^{1,2,3}$, Alexa Hollinger ${ }^{1,2,4}$, Alain Cariou ${ }^{5}$, Nicolas Deye ${ }^{2,6}$, Antoine Vieillard-Baron $^{7,8,9}$, Samir Jaber ${ }^{10}$, Benjamin G. Chousterman ${ }^{1}$, Qin Lu ${ }^{11}$, Pierre François Laterre ${ }^{12}$, Xavier Monnet ${ }^{13}$, Michael Darmon ${ }^{14}$, Marc Leone ${ }^{15}$, Bertrand Guidet ${ }^{16}$, Romain Sonneville ${ }^{17}$, Jean-Yves Lefrant ${ }^{18}$, Marie-Céline Fournier ${ }^{1,2}$, Matthieu Resche-Rigon ${ }^{19}$, Alexandre Mebazaa ${ }^{1,2,3}$, Matthieu Legrand ${ }^{1,2,3^{*}}$, , and On behalf of FROG-ICU investigators

\section{Abstract}

Purpose: Acute kidney injury (AKI) is associated with the activation of the renin-angiotensin system. Whether angiotensin-converting enzyme inhibitors (ACEi) or angiotensin-receptor blockers (ARB) improve outcome in patients recovering from $\mathrm{AKI}$ remains unexplored. The purpose was to investigate the association between prescription of ACEi/ARB at intensive care unit (ICU) discharge and 1-year outcome in patients recovering from AKI.

Methods: Association between ACEi/ARB and 1-year mortality rate was explored in 1551 patients discharged from 21 European ICUs in an observational cohort. One-year all-cause mortality after ICU discharge was the primary endpoint. AKI was defined using the kidney disease improvement global outcome definition. Propensity score matching was used to consider the probability to receive ACEi/ARB at ICU discharge and included chronic heart failure, ACEi/ ARB on ICU admission, Charlson Comorbidity Index, age, diabetes mellitus, chronic kidney disease, estimated glomerular filtration rate and arterial blood pressure at ICU discharge vasopressors and renal replacement therapy.

Results: Overall, 1-year mortality was 28 and 15\% in patients with AKI $(n=611,39 \%)$ and without AKI $(n=940)$, respectively. In patients with $\mathrm{AKI}$, unadjusted, adjusted and propensity-score matched 1-year mortality rates were lower in patients treated with ACEi/ARB at ICU discharge [HR of 0.55 (0.35-0.89), HR of $0.45(0.27-0.75)$, and HR of $0.48(0.27-0.85, p<0.001)$, respectively]. These results were consistent across sensitivity analysis. No association was observed in patients without AKI.

Conclusions: In patients discharged alive from the ICU after experiencing AKI, ACEi/ARB prescription at discharge is associated with a decrease in 1-year mortality.

Trial registration: ClinicalTrials.gov NCT01367093. Registered on 6 June 2011.

Keywords: Angiotensin-converting enzyme inhibitors, Angiotensin-receptor blockers, ICU, Critically III, ICU discharge, Acute kidney injury, Mortality 


\section{Introduction}

Acute kidney injury (AKI) is associated with activation of the renin-angiotensin-aldosterone system (RAAS) [1, 2]. Activation of the RAAS has been shown to be associated with long-term detrimental consequences, especially with chronic kidney disease (CKD) and cardiovascular damage [3-7]. In this line, angiotensin converting enzymes inhibitors (ACEi) and or angiotensin-receptor blockers (ARB) have been recognized as key drugs to protect the kidney and the heart in chronic conditions such as diabetes or heart failure [8]. Protective effects of ACEi/ARB are thought to lie in the prevention of organ fibrosis development $[2,9,10]$. On the other hand, ACEi/ARB have long been considered to be potential nephrotoxic drugs in acute settings. It is currently recommended to stop ACEi/ $A R B$ in the setting of AKI caused by hypovolemia or hypotension [11]. An unresolved question is whether an ACEi or ARB should be started in patients who recovered from AKI. Recently, potential protective effects of ACEi/ $A R B$ following acute injury have, however, been reported $[1,2,9,12]$. The impact of ACEi/ARB in patients recovering from AKI still remains largely unexplored. In this study, we hypothesized that administration of ACEi/ARB in patients that had AKI during their ICU stay would be associated with lower 1-year mortality rate.

\section{Materials and methods}

\section{Patients}

The outcome of patients experiencing AKI and discharged alive from the ICU was explored in the FROGICU cohort (trials.gov identifier: NCT01367093) [13]. The study was conducted in France and Belgium in accordance with Good Clinical Practice (Declaration of Helsinki 2002) and Ethical Committee approvals (Comité de Protection des Personnes-Ile de France IV, IRB no. 00003835 and Commission d'éthique biomédicale hospitalo-facultaire de l'hôpital de Louvain, IRB no. B403201213352). Patients were included from August 2011 to June 2013. The study was an international observational study including 2087 consecutive patients admitted to $21 \mathrm{ICUs}$ receiving mechanical ventilation and/or vasopressors for at least $24 \mathrm{~h}$. The protocol has previously been described elsewhere [14]. Among the 2087 included patients, 1570 (74\%) were discharged alive from ICU and 1551 had data regarding ACEi or ARB treatment available representing our study population.

\section{Definitions of acute kidney injury}

The AKI definition was based on the KDIGO criteria using serum creatinine (Screat) or need for renal replacement therapy [15]. The baseline Screat was ICU admission Screat when eGFR was above $60 \mathrm{~mL} / \mathrm{min} / 1.73 \mathrm{~m}^{2}$ or
In patients discharged alive from the ICU after experiencing AKI, ACEi prescription at discharge is associated with a decrease in oneyear mortality rate.

based on the Modified and Diet Renal Disease formula (MDRD) equation in all other cases. Severe AKI was defined as AKI stage 2 or 3. Patients who had AKI during ICU stay were explored for non-recovery, defined as acute kidney disease (AKD) patients [16]. AKD was defined as Screat level at discharge $>1.5$ times baseline Screat level among patients who developed AKI in the ICU.

\section{Endpoint}

The primary endpoint was 1-year all-cause mortality after ICU discharge. Outcome was collected by questionnaire and/or phone contact and/or civil registry examination.

\section{Statistical analysis}

The association between mortality and ACEi/ARB prescribed at ICU discharge was assessed using both univariate and multivariate analyses after adjustment for potential confounding factors (age, Charlson score, CKD, diabetes mellitus, chronic heart failure, ACEi/ARB intake previous to ICU admission, administration of vasopressors during ICU stay, RRT during ICU stay, systolic blood pressure at ICU discharge). The proportional hazards assumption of the Cox regression was tested as appropriate using the and Therneau approach [17]. Different sensitivity analyses were conducted. In particular, a potential center effect was included in the multivariate mode using a robust estimator of the variance, which takes into account potential intra-center correlation. Missing values were handled by multiple imputation by chained equations [18], and results of the association between the exposure and the main outcome measure after multiple imputation were reported as a sensitivity analysis (e-Table 1). Different variables were also added to the multivariate model: cardiogenic shock as the cause of ICU admission, and the level of NT-proBNP at ICU discharge. The log-linearity of NT-proBNP association with the outcome was checked using restricted cubic spline.

The association between ACEi/ARB at discharge and 1-year outcome was also considered after adjustment in various subgroups: patients treated or not by ACEi/ARB at ICU admission, after exclusion of patients with chronic renal disease, patients with non-severe versus severe AKI (defined by KDIGO class 2 or 3 ) and patients with or without acute kidney disease as previously defined [16].

Finally, the effect of ACEi/ARB on mortality was estimated using propensity score matching (PS-matching). 
Given the observational nature of the data, treatment allocation was not randomly allocated in the study population. The risk of allocation bias due to the presence of confounders was handled using PS-matching., which in our investigation took the probability into account that a patient with specific baseline characteristics had a prescription of ACEi/ARB at ICU discharge, thus allowing the comparison of patients with or without ACEi/ARB at discharge having similar characteristics. PS-matching characteristics included chronic heart failure, ACEi/ARB on ICU admission, Charlson's score, eGFR at discharge (using the MDRD formula), systolic blood pressure at discharge, age, diabetes, CKD, and vasopressors or RRT during ICU stay. Variables included in the propensity score were selected when either major difference among treated and non-treated patients were observed or when they were identified as potentially true confounded (i.e. associated with both treatment allocation and prognosis). Each patient treated with ACEi was matched to one untreated control with similar PS using the nearest-/ARB neighbor approach, with no replacement and a calliper size of 0.2 . Imbalance between treated and untreated patients before and after PS matching was assessed using a standardized difference, considering less than $10 \%$ acceptable to define the study patients' characteristics balanced with respect to the previously described features. The association between outcome and ACEi/ARB prescription was further described in patients with or without AKD.

\section{Table 1 Characteristics of patients included in patients with or without AKI and discharge alive from intensive care unit (ICU)}

\begin{tabular}{|c|c|c|c|c|}
\hline & $\%$ of missingness & Patients without AKI $(n=940)$ & Patients with AKI $(n=611)$ & $p$ value \\
\hline Age (years) & $0(0 \%)$ & $58(46 ; 69)$ & $67(55 ; 76)$ & $<0.0001$ \\
\hline Male gender (\%) & $0(0 \%)$ & $586(62.3)$ & $396(65)$ & 0.32 \\
\hline $\mathrm{BMI}\left(\mathrm{kg} / \mathrm{m}^{2}\right)$ & $579(37 \%)$ & $25.3(22.3 ; 28.7)$ & $27.8(24.4 ; 32.5)$ & $<0.0001$ \\
\hline Charlson score & $0(0 \%)$ & $2(0 ; 4)$ & $4(2 ; 5)$ & $<0.0001$ \\
\hline SAPS-II & $1(<1 \%)$ & $41(31 ; 54)$ & $53(41 ; 67)$ & $<0.0001$ \\
\hline SOFA at admission & $572(37 \%)$ & $5(3 ; 7)$ & $8(6 ; 11)$ & $<0.0001$ \\
\hline Cause of admission & $0(0 \%)$ & & & $<0.0001$ \\
\hline Septic shock & & $148(15.7 \%)$ & $185(30 \%)$ & \\
\hline Acute respiratory failure & & $210(22.3 \%)$ & $90(15 \%)$ & \\
\hline Cardiogenic shock & & $44(4.7 \%)$ & $61(10 \%)$ & \\
\hline Hemorrhagic shock & & $35(3.7 \%)$ & $48(8 \%)$ & \\
\hline $\mathrm{OHCA}$ & & $73(7.8 \%)$ & $44(7 \%)$ & \\
\hline \multicolumn{5}{|l|}{ Medical history } \\
\hline Diabetes mellitus & $\mathrm{O}(0 \%)$ & $115(12.2 \%)$ & $154(25 \%)$ & $<0.0001$ \\
\hline Chronic heart failure & $0(0 \%)$ & $38(4 \%)$ & $68(11 \%)$ & $<0.0001$ \\
\hline Chronic kidney disease & $0(0 \%)$ & $21(2 \%)$ & $140(23 \%)$ & $<0.0001$ \\
\hline Hypertension & $0(0 \%)$ & $295(31.4 \%)$ & $340(56 \%)$ & $<0.0001$ \\
\hline COPD & $0(0 \%)$ & $95(10.1 \%)$ & $75(12 \%)$ & 0.18 \\
\hline RASi at admission & $12(<1 \%)$ & $183(19.6 \%)$ & $210(35 \%)$ & $<0.0001$ \\
\hline \multicolumn{5}{|l|}{ During ICU stay } \\
\hline In-ICU LOS (days) & $0(0 \%)$ & $11(7 ; 19)$ & $14(8 ; 24)$ & $<0.0001$ \\
\hline Tracheotomy & $0(0 \%)$ & $159(16.9)$ & $81(13 \%)$ & 0.052 \\
\hline Inotrope/vasopressor & $0(0 \%)$ & $621(66.1)$ & $520(85 \%)$ & $<0.0001$ \\
\hline $\mathrm{RBC}$ & $0(0 \%)$ & $318(33.8)$ & $349(57 \%)$ & $<0.0001$ \\
\hline \multicolumn{5}{|l|}{ Status at discharge } \\
\hline $\mathrm{SBP}(\mathrm{mmHg})$ & $197(13 \%)$ & $123(111 ; 137)$ & $128(112 ; 142)$ & 0.0071 \\
\hline $\mathrm{DBP}(\mathrm{mmHg})$ & $255(16 \%)$ & $69(60 ; 77)$ & $67(57 ; 76)$ & 0.011 \\
\hline eGFR $\left(\mathrm{ml} / \mathrm{min} / 1.73 \mathrm{~m}^{2}\right)$ & $142(9 \%)$ & $104.6(91.1 ; 118.8)$ & $52.6(27.2 ; 85)$ & $<0.0001$ \\
\hline $\mathrm{ACEi}$ & $0(0 \%)$ & $129(14 \%)$ & $109(18 \%)$ & 0.028 \\
\hline RASi introduction & $12(<1 \%)$ & $59(6.3 \%)$ & $35(5.7 \%)$ & 0.67 \\
\hline 1-year mortality & $0(0 \%)$ & $147(15.6 \%)$ & $173(28 \%)$ & $<0.0001$ \\
\hline
\end{tabular}

Data are expressed as median with interquartile range (in brackets) or count with percentage (in parentheses) as appropriate BMI body mass index, SAPS2 simplified acute physiology score 2, SOFA sequential organ failure assessment, COPD chronic obstructive pulmonary disease, eGFR estimated glomerular filtration rate, $R B C$ red blood cells, $S B P$ systolic blood pressure, $D B P$ diastolic blood pressure, $O H C A$ out-hopsital cardiac arrest, $A C E i$ angiotensinconverting enzyme inhibitors 
Table 2 Comparison of AKI patients' characteristics according to the treatment at ICU discharge

\begin{tabular}{|c|c|c|c|}
\hline & No ACEi/ARB at discharge $(n=502)$ & ACEi/ARB at discharge $(n=109)$ & $p$ value \\
\hline Age (years) & $66(53.2 ; 76)$ & $70(61 ; 80)$ & 0.0083 \\
\hline Male gender (\%) & $330(66 \%)$ & $66(61 \%)$ & 0.3 \\
\hline $\mathrm{BMI}\left(\mathrm{kg} / \mathrm{m}^{2}\right)$ & $27.8(24.4 ; 32.2)$ & $28.1(24.6 ; 32.6)$ & 0.85 \\
\hline Charlson score & $4(2 ; 5)$ & $4(3 ; 6)$ & 0.005 \\
\hline SAPS- $\|$ & $54(41 ; 66.8)$ & $53(41 ; 73)$ & 0.37 \\
\hline SOFA at admission & $8(6 ; 11)$ & $8(5 ; 10)$ & 0.32 \\
\hline Cause of admission & & & 0.005 \\
\hline Septic shock & $159(31.7)$ & $26(24 \%)$ & \\
\hline Acute respiratory failure & $67(13 \%)$ & $23(21 \%)$ & \\
\hline Cardiogenic shock & $45(9 \%)$ & $16(15 \%)$ & \\
\hline Hemorrhagic shock & $44(9 \%)$ & $4(4 \%)$ & \\
\hline OHCA & $31(6 \%)$ & $13(12 \%)$ & \\
\hline \multicolumn{4}{|l|}{ Medical history } \\
\hline Diabetes mellitus & $116(23 \%)$ & $38(35 \%)$ & 0.01 \\
\hline Chronic heart failure & $53(11 \%)$ & $15(14 \%)$ & 0.34 \\
\hline Chronic kidney disease & $115(23 \%)$ & $25(23 \%)$ & 1 \\
\hline Hypertension & $274(55 \%)$ & $66(61 \%)$ & 0.26 \\
\hline COPD & $54(11 \%)$ & $21(19 \%)$ & 0.014 \\
\hline RASi at admission & $138(28 \%)$ & $72(67 \%)$ & $<0.0001$ \\
\hline \multicolumn{4}{|l|}{ During ICU stay } \\
\hline In-ICU LOS (days) & $14(8 ; 25)$ & $12(8 ; 21)$ & 0.21 \\
\hline Tracheotomy & $68(14 \%)$ & $13(12 \%)$ & 0.65 \\
\hline Inotrope/vasopressor & $436(87 \%)$ & $84(77 \%)$ & 0.0093 \\
\hline $\mathrm{RBC}$ & $285(57 \%)$ & $64(59 \%)$ & 0.71 \\
\hline \multicolumn{4}{|l|}{ Status at discharge } \\
\hline $\mathrm{SBP}(\mathrm{mmHg})$ & $127(111 ; 140)$ & $134(116 ; 146)$ & 0.052 \\
\hline $\mathrm{DBP}(\mathrm{mmHg})$ & $66.5(57 ; 76)$ & $68.5(58.2 ; 76.8)$ & 0.47 \\
\hline eGFR $\left(\mathrm{ml} / \mathrm{min} / 1.73 \mathrm{~m}^{2}\right)$ & $51.8(27 ; 85.4)$ & $55.2(27.6 ; 83.2)$ & 0.84 \\
\hline RASi introduction & $0(0 \%)$ & $35(32.7 \%)$ & $<0.0001$ \\
\hline 1-year mortality & $153(31 \%)$ & $20(18 \%)$ & 0.011 \\
\hline
\end{tabular}

Data are expressed as median with with interquartile range (in brackets) or count with percentage (in parentheses) as appropriate

$B M I$ body mass index, SAPS2 simplified acute physiology score 2, SOFA sequential organ failure assessment, COPD chronic obstructive pulmonary disease, eGFR estimated glomerular filtration rate, $R B C$ red blood cells, $S B P$ systolic blood pressure, $D B P$ diastolic blood pressure, $O H C A$ out-hopsital cardiac arrest, $A C E i$ angiotensinconverting enzyme inhibitors

Data are expressed in median with interquartile range or count with percentage as appropriate. $p<0.05$ was considered statistically significant. All analyses were performed using R statistical software (The "R" Foundation for Statistical Computing, Vienna, Austria, URL http:// www.jstatsoft.org/v42/i08/).

\section{Results}

\section{Patients characteristics}

A total of 1551 patients survived during their ICU stay, of which 611 (39\%) had an AKI episode during the ICU stay. Table 1 details patient characteristics at discharge according to outcome and Table 2 details patient characteristics according to treatment at discharge. Among patients who developed AKI during ICU stay, 186 were classified as AKI stage 1, 94 as stage 2, and 331 patients as stage 3. Overall, 1-year mortality of the AKI group was $28 \%(n=173)$. The rate of ACEi/ARB introduction was slightly higher in post-AKI patients than in patients without AKI (6.3 vs. $5.7 \%)$.

\section{Impact of ACEi/ARB prescription on outcome}

Patients prescribed with ACEi/ARB at ICU discharge $[n=109(18 \%)]$ revealed a lower mortality rate compared to those who were not ( 18 vs $.31 \%$ respectively, $p=0.01$; Fig. 1). Unadjusted mortality risk was greater in patients untreated when compared to those that were prescribed with ACEi/ARB at ICU discharge. Mortality risk remained significantly associated to non-prescription of ACEi/ARB after adjustment for prognostic variables (Fig. 2). 


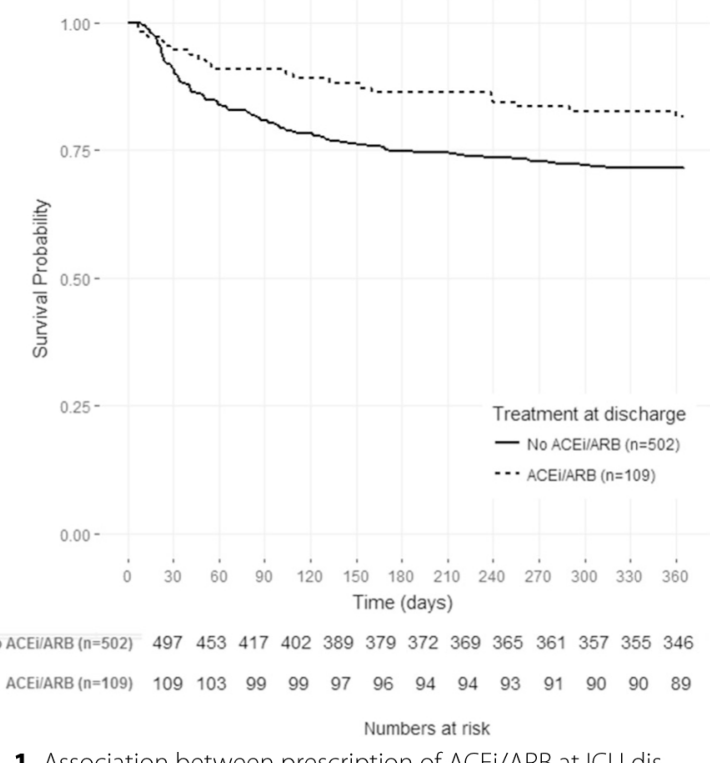

Fig. 1 Association between prescription of ACEi/ARB at ICU discharge and 1-year survival in AKI patients; association between ACEi/ ARB at intensive care unit discharge and 1-year mortality unadjusted

Propensity-based matching produced 82 matched pairs with standardized differences in patient characteristics of less than $10 \%$, indicating a successful balance of potential confounders between treated and untreated patients (e-Table 2; e-Fig. 1). SAPS2 score was 55 (44-73) in patients treated with ACEi at discharge and 56 (40-68) in patients not treated. PS analysis confirmed the suggested protective effect of ACEi on 1-year mortality (18 vs. $35 \%, p=0.01$ ). In the PS-matched patients, 1-year mortality was lower in patients receiving ACEi compared to those who did not. All sensitivity analysis (including cluster effect, multiple imputation, NT-ProBNP at discharge and cardiogenic shock) showed consistent effects on the association between ACEi/ ARB and outcome (Fig. 2). Associations between outcome and ACEi/ARB in sub-goups are presented in Fig. 2.

In contrast, mortality in ACEi and non-ACEi subgroups was similar in non-AKI patients in both adjusted analysis and PS matching [HR of 1.8 (1.21-2.68), HR of $0.87(0.53-1.42)$, and HR of $1.00(0.58-1.73)$ respectively; eFig. 2].

\section{Impact of AKI severity and ACEi prescription on outcome}

Among patients who experienced AKI, Figs. 2 and 3 show that benefits associated with ACEi treatment for 1-year mortality may be influenced by AKI severity or kidney function recovery at discharge. ACEi were associated with benefits on 1-year mortality in patients with both, high and low eGFR at discharge. When considering AKI severity, the association was found significant in non-severe AKI and in patients with AKD.

\section{Discussion}

In this ancillary study of FROG-ICU which enrolled the widest population of ICU patients at discharge, we observed an association between the prescription of ACEi/ARB at ICU discharge and 1-year survival in patients with AKI. Propensity analysis confirmed the suggested protective effect. This association suggests that treatment with ACEi in this setting might improve outcome after AKI.

Acute kidney injury is associated with worse longterm outcome in many ICU studies [19]. The reasons for such association remain largely unknown, but the impact of AKI on remote organ function and damage has been highlighted [20]. Activation of the RAAS triggers vasoconstriction and pro-fibrotic pathways involved in chronic organ damage and dysfunction [3, 9, 21, 22]. Blocking the RAAS using ACEi/ARB is currently recommended to prevent occurrence or heart damage and failure after acute myocardial infarction or to limit progression of chronic heart failure [19]. ACEi/ARB are also recommended for patients with diabetic nephropathy, hypertension and proteinuria to limit the progression towards CKD [23].

AKI leads to activation of the RAAS and may therefore lead to systemic chronic cardiovascular and renal damage. In this regard, AKI promotes renal fibrosis and chronic kidney damage [3]. AKI was further perceived to be a risk factor for chronic kidney disease and to be associated with a high incidence of cardiovascular complications [22]. While ACEi/ARB administration in chronic conditions is beneficial, they have, however, long been considered as potential nephrotoxic in altered intra-renal hemodynamics in acute condition. ACEi/ARB may buffer renal autoregulation and impair glomerular filtration rate in patients with systemic hypotension or hypovolemia [11], this is not, however, associated with kidney damage.

Acute kidney injury further carries a high risk of longterm mortality and cardiovascular events. Several pre-clinical data suggest that AKI may induce remote cardiovascular damage, which may partially be causal in the long-term outcome after AKI [16, 20]. Furthermore, AKI, chronic kidney disease and chronic heart failure are thought to share common pathophysiological pathways, including activation of the renin-angiotensin-aldosterone system and activation of pro-fibrotic pathways [21, 24]. Altogether, previous pre-clinical and clinical studies suggest that AKI activates the renin-angiotensin-aldosterone system and may promote the development of chronic renal and cardiac injuries through organs fibrosis. It is highly plausible that protective strategies targeting these pathways may improve long-term outcome after AKI. We therefore hypothesized that ACEi/ ARB may prevent long-term consequences of AKI. 


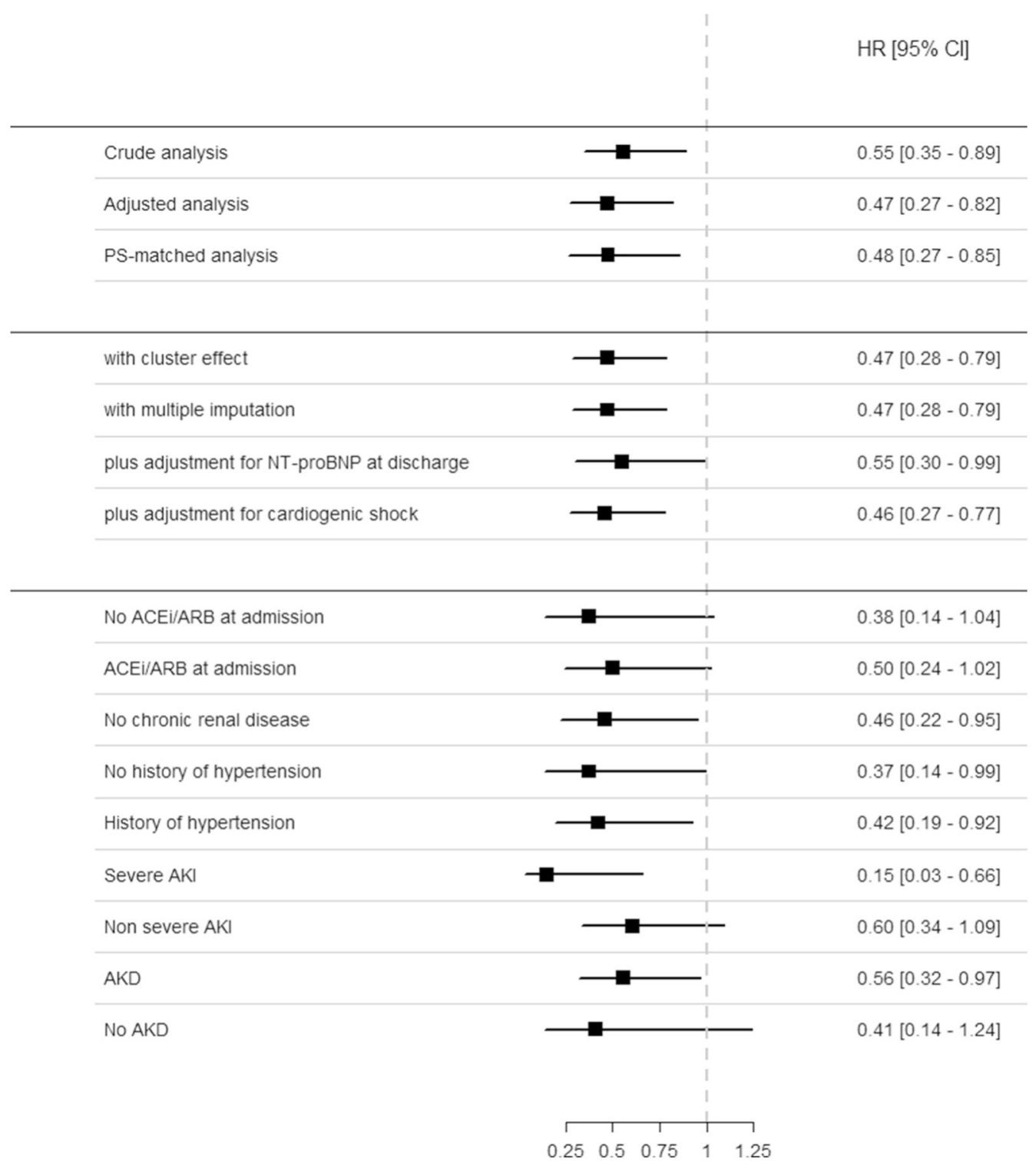

Fig. 2 Association between ACEi/ARB prescription and outcome of patients with acute kidney injury in crude analysis, after adjustment and propensity score (PS) matching, in sensitivity analysis and in subgroups. AKI acute kidney injury, AKD acute kidney disease, HR hazard ratio (confidence interval 95\%)

We acknowledge that this study suffers from several limitations. First, the observational nature of the data prevents the confirming of causality even though the association was also observed after adjustment for confounding variables and using PS-matching. We must, however, acknowledge that residual confounding factors may persist. This reinforces the need for a randomized controlled trial. The data, however, do not suggest any harm, and provide solid information for further interventional randomized controlled trials. Secondly, the exact date of ACEi/ARB initiation and patient compliance after ICU discharge was not controlled in the study, and the introduction or interruption of treatment after ICU discharge was not available or controlled. Third, the dose ACEi was not recorded. Even though the cohort was large, the sample size of patients treated with ACEi/ARB at discharge may limit power for sub-groups analysis. Urine output and true baseline Screat were not available in this cohort. Acute kidney disease was also assessed at ICU discharge before 3 months of follow-up in most cases, which may lead to under-evaluation of recovery. Also, kidney and heart function was not assessed during the follow-up, and therefore the impact of ACEi-induced 


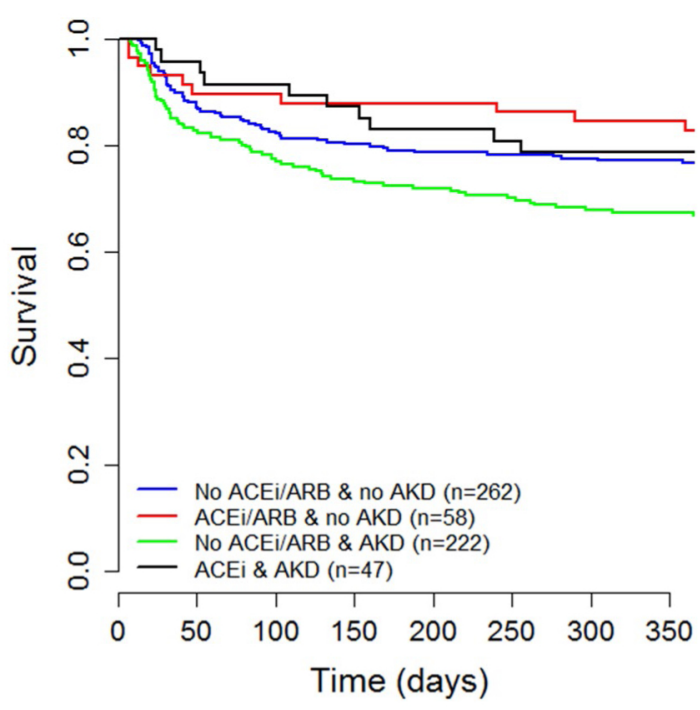

Fig. 3 Association between prescription of ACEi/ARB at ICU discharge and 1-year survival in AKI patients according the renal recovery; association between ACEI/ARB at intensive care unit discharge and 1-year mortality unadjusted in patients with or without acute kidney disease (AKD)

cardiovascular and renal disease prevention on the outcome could not be confirmed. Finally, as the causes of death after ICU are not known in FROG-ICU, it was unfortunately not possible to show a decrease in the incidence of cardiovascular or renal deaths between exposed and unexposed populations.

However, whatever the patient compliance, the dose and the effects on different organs, we report a reduction in 1-year mortality. This main result could encourage the performing of a large randomized study in ICU patients experiencing an AKI during ICU stay.

\section{Conclusion}

To conclude, the results of this study suggest that ACEi/ $\mathrm{ARB}$ may be considered as a preventive strategy for longterm outcome for patients discharged alive from ICU, after having experienced an episode of AKI. Whether ACEi/ARB could prevent chronic organ damage and ultimately improve outcome in patients recovering from AKI needs to be properly validated in further randomized controlled clinical trials.
${ }^{3}$ University Paris Diderot, 75475 Paris, France. ${ }^{4}$ Department of Anesthesia, Surgical Intensive Care, Prehospital Emergency Medicine and Pain Therapy, University Hospital Basel, Basel, Switzerland. ${ }^{5}$ Medical Intensive Care Unit, Cochin University Hospital, Assistance Publique-Hôpitaux de Paris, Paris, France. ${ }^{6}$ Medical and Toxicological Intensive Care Unit, Lariboisière Hospital, Université Paris Sorbonne Cité, Paris Diderot, Assistance Publique des Hôpitaux de Paris, Paris, France. ${ }^{7}$ Intensive Care Unit, University Hospital Ambroise Paré, Assistance Publique-Hopitaux de Paris, 26930 Boulogne-Billancourt, France. ${ }^{8}$ INSERM U-1018, CESP, Team 5, University of Versailles Saint-Quentin en Yvelines, Villejuif, France. ${ }^{9}$ Faculty of Medicine Paris Ile-de-France Ouest, University of Versailles Saint-Quentin en Yvelines, Saint-Quentin en Yvelines, France. ${ }^{10}$ Intensive Care Unit, Anaesthesia and Critical Care Department, Saint Eloi Teaching Hospital, Centre Hospitalier Universitaire Montpellier, Montpellier University, Montpellier, France. ${ }^{11}$ Multidisciplinary Intensive Care Unit, Department of Anesthesiology and Critical Care Medicine, La Pitié-Salpêtrière Hospital, Assistance Publique Hôpitaux de Paris, UPMC Paris 6, Paris, France.

12 Department of Critical Care Medicine, Saint Luc University Hospital, Université Catholique de Louvain, Avenue Hippocrate 10, 1200 Brussels, Belgium.

${ }^{13}$ Medical Intensive Care Unit, Bicêtre Hospital, Paris-Sud University Hospitals, Inserm UMR_S999, Paris-Sud University, Le Kremlin-Bicêtre, France. ${ }^{14}$ Medical ICU, St-Louis Hospital, Université Paris Sorbonne Cité, Paris Diderot, Assistance Publique des Hôpitaux de Paris, Paris, France. ${ }^{15}$ Service d'anesthésie et de réanimation, Hôpital Nord, Assistance Publique-Hôpitaux de Marseille, Aix Marseille Université, Marseille, France. ${ }^{16}$ Service de Réanimation Médicale, Hôpital Saint-Antoine, Assistance Publique-Hôpitaux de Paris, Sorbonne Université, Paris, France. ${ }^{17}$ Medical ICU, Bichat Hospital, Université Paris Sorbonne Cité, Paris Diderot, Assistance Publique des Hôpitaux de Paris, Paris, France. ${ }^{18}$ Department of Anesthesiology, Critical Care, Emergency and Pain Medicine, Nimes University Hospital, 30029 Nîmes, France. ${ }^{19}$ ECSTRA Team, INSERM, Service de biostatistique et information Médicale, Hôpitaux Universitaires St-Louis-Lariboisière, Assistance Publique-Hôpitaux de Paris, Université Paris Diderot-Paris 7, Sorbonne Paris Cité, Paris, France.

\section{Acknowledgements}

The authors are particularly grateful to CRAs and healthcare providers of all the investigating centers. We also thank the Centre de Recherche Clinique (CRC) of Lariboisière University Hospital for his support.

\section{Author contribution}

Study concept and design: Gayat, Legrand, Mebazaa; Acquisition of data: Gayat, Mebazaa, Vieillard-Baron, Cariou, Deye, Jaber, Chousterman, Lu, Laterre, Monnet, Leone, Guidet, Lefrant, Fournier, Legrand; Analysis and interpretation of data: Gayat, Legrand, Mebazaa; Drafting of the manuscript: Legrand, Gayat, Hollinger, Mebazaa; Critical revision of the manuscript for important intellectual content: all declared authors; Statistical analysis: Gayat; Obtained funding: Gayat, Mebazaa; Administrative, technical, or material support: Gayat, Mebazaa, Fournier; Study supervision: Gayat, Legrand, Mebazaa; All authors have read and approved the final manuscript.

\section{Funding}

FROG-ICU was funded by the Programme Hospitalier de la Recherche Clinique (AON 10-216) and by a research grant from the Société Française d'Anesthésie_Réanimation.

\section{Complaince with ethical standards}

\section{Conflicts of interest}

EG received research grants from Sphingotec, and consultancy fees from Magnisense and Roche Diagnostics. AM received speaker's honoraria from Abbott, Novartis, Orion, Roche and Servier, and fees as a member of the advisory board and/or Steering Committee from Cardiorentis, Adrenomed, MyCartis, Neurotronik and Sphyngotec. ML received research grants from Sphingotec, consultancy fees from Astellas and Lecture fees from Gilead and Fresenius. The remaining authors declare that they have no competing interests.

\section{Author details}

${ }^{1}$ Department of Anaesthesiology, Critical Care Medicine and Burn Unit, Saint Louis and Lariboisière University Hospitals, AP-HP, 2 rue A. Paré, 75010 Paris, France. ${ }^{2}$ Institut National de la Santé et de la Recherche Médicale (INSERM), Lariboisière Hospital and INI-CRCT Network, INSERM UMR-S942, Paris, France. 


\section{References}

1. Cheng S-Y, Chou Y-H, Liao F-L et al (2016) Losartan reduces ensuing chronic kidney disease and mortality after acute kidney injury. Sci Rep 6:34265. https://doi.org/10.1038/srep34265

2. Efrati S, Berman S, Hamad RA et al (2012) Effect of captopril treatment on recuperation from ischemia/reperfusion-induced acute renal injury. Nephrol Dial Transplant 27:136-145. https://doi.org/10.1093/ndt/gfr256

3. Burrell LM, Burchill L, Dean RG et al (2012) Chronic kidney disease: cardiac and renal angiotensin-converting enzyme (ACE) 2 expression in rats after subtotal nephrectomy and the effect of ACE inhibition. Exp Physiol 97:477-485. https://doi.org/10.1113/expphysiol.2011.063156

4. Chou Y-H, Huang T-M, Pan S-Y et al (2017) Renin-angiotensin system inhibitor is associated with lower risk of ensuing chronic kidney disease after functional recovery from acute kidney injury. Sci Rep 7:46518. https://doi.org/10.1038/srep46518

5. Horkan CM, Purtle SW, Mendu ML et al (2015) The association of acute kidney injury in the critically ill and postdischarge outcomes: a cohort study. Crit Care Med 43:354-364. https://doi.org/10.1097/CCM.00000 00000000706

6. Parr SK, Matheny ME, Abdel-Kader K et al (2017) Acute kidney injury is a risk factor for subsequent proteinuria. Kidney Int. https://doi. org/10.1016/j.kint.2017.07.007

7. Sawhney S, Marks A, Fluck N et al (2017) Acute kidney injury as an independent risk factor for unplanned 90 -day hospital readmissions. BMC Nephrol 18:9. https://doi.org/10.1186/s12882-016-0430-4

8. Rana I, Velkoska E, Patel SK et al (2015) MicroRNAs mediate the cardioprotective effect of angiotensin-converting enzyme inhibition in acute kidney injury. Am J Physiol Renal Physiol 309:F943-F954. https://doi. org/10.1152/ajprenal.00183.2015

9. Evans M, Carrero J-J, Szummer K et al (2016) Angiotensin-converting enzyme inhibitors and angiotensin receptor blockers in myocardial infarction patients with renal dysfunction. J Am Coll Cardiol 67:16871697. https://doi.org/10.1016/j.jacc.2016.01.050

10. Burchill L, Velkoska E, Dean RG et al (2008) Acute kidney injury in the rat causes cardiac remodelling and increases angiotensin-converting enzyme 2 expression. Exp Physiol 93:622-630. https://doi.org/10.1113/ expphysiol.2007.040386

11. Joannidis M, Druml W, Forni LG et al (2017) Prevention of acute kidney injury and protection of renal function in the intensive care unit: update 2017: expert opinion of the Working Group on Prevention, AKI section, European Society of Intensive Care Medicine. Intensive Care Med 43:730-749. https://doi.org/10.1007/s00134-017-4832-y

12. Cheungpasitporn W, Thongprayoon C, Srivali N et al (2015) Preoperative renin-angiotensin system inhibitors use linked to reduced acute kidney injury: a systematic review and meta-analysis. Nephrol Dial Transplant 30:978-988. https://doi.org/10.1093/ndt/gfv023
13. Gayat E, Cariou A, Deye N et al (2018) Determinants of long-term outcome in ICU survivors: results from the FROG-ICU study. Crit Care Lond Engl 22:8. https://doi.org/10.1186/s13054-017-1922-8

14. Mebazaa A, Casadio MC, Azoulay E et al (2015) Post-ICU discharge and outcome: rationale and methods of the The French and euRopean Outcome reGistry in Intensive Care Units (FROG-ICU) observational study. BMC Anesthesiol 15:143. https://doi.org/10.1186/s12871-015-0129-2

15. Hoste EAJ, Bagshaw SM, Bellomo R et al (2015) Epidemiology of acute kidney injury in critically ill patients: the multinational AKI-EPI study. Intensive Care Med 41:1411-1423. https://doi.org/10.1007/s00134015-3934-7

16. Forni LG, Darmon M, Ostermann M et al (2017) Renal recovery after acute kidney injury. Intensive Care Med 43:855-866. https://doi.org/10.1007/ s00134-017-4809-x

17. Grambsch P, TherneauT (1994) Proportional hazards tests and diagnostics based on weighted residuals. Biometrika 81:515-526

18. Harrell FE, Lee KL, Mark DB (1996) Multivariable prognostic models: issues in developing models, evaluating assumptions and adequacy, and measuring and reducing errors. Stat Med 15:361-387. https://doi.org/10.1002/ (SICI) 1097-0258(19960229)15:4\%3c361:AID-SIM168\%3e3.0.CO:2-4

19. Prescott HC, Angus DC (2018) Enhancing recovery from sepsis: a review. JAMA 319:62-75. https://doi.org/10.1001/jama.2017.17687

20. Dépret F, Prud'homme M, Legrand M (2017) A role of remote organs effect in acute kidney injury outcome. Nephron 137:273-276. https://doi. org/10.1159/000476077

21. Simões E, Silva AC, Teixeira MM (2016) ACE inhibition, ACE2 and angiotensin-(1-7) axis in kidney and cardiac inflammation and fibrosis. Pharmacol Res 107:154-162. https://doi.org/10.1016/j.phrs.2016.03.018

22. Zhong J, Basu R, Guo D et al (2010) Angiotensin-converting enzyme 2 suppresses pathological hypertrophy, myocardial fibrosis, and cardiac dysfunction. Circulation 122:717-728. https://doi.org/10.1161/circulatio naha.110.955369 (18 p following 728)

23. Whelton PK, Carey RM, Aronow WS et al (2017) 2017 ACC/AHA/AAPA/ ABC/ACPM/AGS/APhA/ASH/ASPC/NMA/PCNA guideline for the prevention, detection, evaluation, and management of high blood pressure in adults: a report of the American College of Cardiology/American Heart Association task force on clinical practice guidelines. Hypertens Dallas Tex. https://doi.org/10.1161/HYP.0000000000000065

24. Chawla LS, Eggers PW, Star RA, Kimmel PL (2014) Acute kidney injury and chronic kidney disease as interconnected syndromes. N Engl J Med 371:58-66. https://doi.org/10.1056/NEJMra1214243 